\title{
Integrative Knowledge Management in Interdisciplinary Research Clusters
}

\author{
http://dx.doi.org/10.3991/ijac.v8i3.4854 \\ Claudia Jooß, Thomas Thiele, René Vossen, Anja Richert, and Sabina Jeschke \\ IMA/ZLW \& IfU, RWTH Aachen University, Aachen, Germany
}

\begin{abstract}
Interdisciplinary Research (IDR) is described as a specific mode of collaboration: Besides the clash of different institutional cultures (e.g. different expectations/working processes), there is a clash of epistemic cultures (i.e. styles of thinking, different languages) [1]. Former research shows that the involved researchers demand an integrative knowledge management to support the expected integration of cultures [1]. In this paper two major aspects regarding integrative knowledge management for IDR are discussed. On the one hand the need for integrative knowledge management based on the researchers' perspective is depicted in the context of a use case. On the other hand the concept of a virtual mean is elaborated, which supports these needs. Both aspects underline the importance of a processaccompanying support in close coordination with the respective needs of the involved researchers.
\end{abstract}

Index Terms - Interdisciplinary Research (IDR), Integration, Knowledge Management, Terminology-based Interfaces

\section{INTRODUCTION}

Advocates of interdisciplinary research (IDR) argue that this special form of research collaboration has a positive influence on problem solving and knowledge production $[2 ; 3 ; 4 ; 5 ; 6]$. IDR is considered to meet today's complex problems - whether the motives derive from innerscientific needs or society. Despite the argument of problem solving and knowledge production, advocates of IDR also point out challenges of the expected integration. Although terms of interdisciplinarity vary and expectations of this form of collaboration are heterogeneous, a consensus shows within the international discourse. This consensus displays integration as a central feature of interdisciplinarity [1]. The National Academies, for instance, state that interdisciplinarity is achieved only if an integration and a synthesis of ideas and methods take place [7]. A combination of concepts, theories, and methods is expected [8;9]. This means that gained insights are not only accumulated ex post, but are also integrated during the cooperation process by means of an overall view. Thus, "synergies" are expected to be formed [10]. Strina describes synergies as interaction of different powers, elements, and parts that, hence, allow more than would be possible due to the features of the single parts [11]. Even though the researchers involved have a significant role due to these conditions $[4 ; 5 ; 12]$, the characteristics and assessments of the researchers involved in interdisciplinary research networks are hardly investigated. We believe that this is an important gap to address, since it is the individual researcher who has to engage in research collaborations and develop scientific results [5]. Moreover, it is the individual re- searcher who is supposed to make an innovative contribution to solve today's complex problems. In contrast, the focus on the individual researchers' perspective provides wide-ranging implications for the design and effectiveness of interdisciplinary collaborations.

Against this background, different researchers of a complex interdisciplinary research cluster have been examined from various perspectives over an investigation period of five years [1]. A central result of this study shows that the researchers involved demand a knowledge management approach to support the expected integration. This so-called integrative knowledge management is a specific knowledge management in terms of the interdisciplinary vision. It comprises the continuous support of synchronization and adaption of the individual research activities with regard to the vision [1]. Integrative describes the assumption that a combination of various measures in a process-accompanying manner are of importance. These are developed, implemented and evaluated in a use case of an interdisciplinary research cluster (cf. Fig. 1) and can be grouped e.g. in virtual and physical measures.

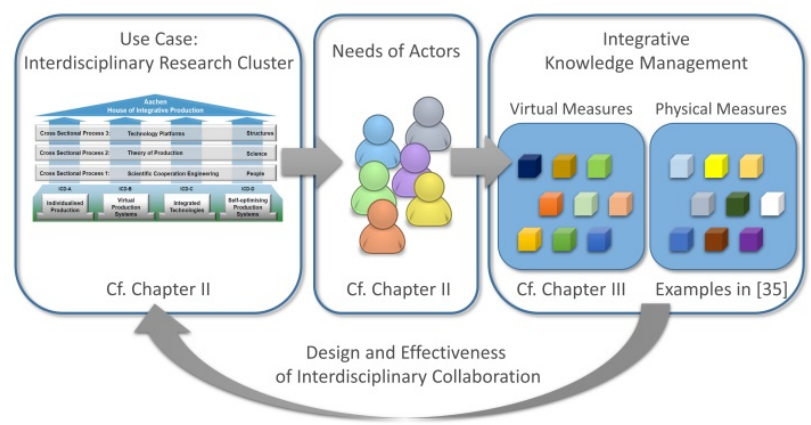

Figure 1. Structure of the paper

Based on this concept the question arises, which needs have to be fulfilled by the depicted measures in order to achieve an interdisciplinary integration.

As primary aim, this paper pursues the research question: Which needs of an integrative knowledge management are required from the researchers' perspective? To do so, chapter II describes both the choice of the research field, the research design and method as well as central results regarding needs from the involved researchers. The second aim of this paper is to present an exemplary virtual mean to support integrative knowledge management. Within the interdisciplinary research cluster, our object of investigation, the involved researchers demand a mean to initiate the exchange on acquiring a common understanding of terminologies. Thus, we outline a concept of a technical solution in the third part of the paper. 


\section{The NEED OF INTEGRATIVE KNOWLEDGE MANAGEMENT IN INTERDISCIPLINARY RESEARCH CLUSTERS}

\section{A. Choice of Research Field and Organizational Structure}

For our single case analysis, we selected an interdisciplinary research cluster as our object of investigation: The Cluster of Excellence (CoE) "Integrative Production Technology for High-Wage countries" at RWTH Aachen University. It was initiated by the German Research Foundation (DFG) and the German Council of Science and Humanities (WR) as part of the German excellence initiative. The consortium is located in Aachen and investigates the resolution of the polylemma of production [13] with various interdisciplinary partners from different faculties of RWTH Aachen University. The objective comprised ways to solve the tradeoff between scale and scope and between plan and value oriented production [14]. Within this CoE, many researchers from different university institutes, associated institutes, further non-university research institutions as well as different industrial and scientific advisors do research on a common vision. The aim is to develop a holistic theory of production by means of integrating economic, ecological, and social aspects. The CoE (cf. Fig. 2 as an overview of its structure) consists of twelve subprojects with about 180 researchers and 200 student assistants in total. Various scientific disciplines (mechanical engineering, material science, mathematics, business studies, communication science, computer science and psychology) are brought together by these researchers who have also obtained various degrees as far as their academic education is concerned. A common understanding of e.g. terminology, language, methods, competences and perceptions of success is needed in order to enable these diverse personnel to cooperate. For overcoming these challenges of interdisciplinary cooperation and for supporting the performance of interdisciplinary research consortia in general new approaches are needed in order to cross link the amount of different researchers and institutes and to transfer solutions between them [15].

Next to several collaborative projects from the field of production technology, the $\mathrm{CoE}$ therefore additionally comprises cross-sectional projects entrusted with this task, the "Cross Sectional Processes" (CSPs). Their task is to design, implement and constantly evaluate concepts of supporting the integration of the different disciplines into the CoE on a physical and virtual level $[15 ; 16]$.

\section{B. Research Gap and Research Design}

Within the framework of this continuous evaluation within the CSPs we draw on a research gap. It is perceived that researchers take on a special role within the integration but they have not been interrogated about their perception yet [1]. The majority of empirical studies on IDR are based on bibliometric data $[3 ; 5 ; 7 ; 9 ; 17 ; 18 ; 19 ; 20 ; 21]$. This database forms the main core for evaluating the design and effectiveness of interdisciplinary collaborations. This aspect, however, is increasingly challenged with criticism within the current discourse. Millar, for instance, claims it is about time to interrogate the researchers involved as well as to investigate how this form of collaboration affects the cooperation process [22]. Long-term studies, thus, aim at gathering insights on how to develop and design the dynamic and complex cooperation processes [12].

Our research contributes to this need by the use of a specific research design. It aims to obtain a view as extensive as possible on the heterogeneous researchers involved in interdisciplinary collaboration. Thus, the $\mathrm{CoE}$ has been examined from various perspectives over an investigation period of five years (2009-2013). In order to distinguish our study from quantitative procedures, neither antecedent nor theoretical concepts are examined, but rather concepts on the researchers' perception of interdisciplinary collaboration are reconstructed from the qualitative data [23]. We advocate the use of different datasets. In order to depict a sample that is as heterogeneous as possible following the principle of maximum structural variation, different researchers have been interrogated across all hierarchical levels in the course of the data collection process:

- Dataset 1: structured guideline-based interviews

- Dataset 2: partially standardized employee survey

- Dataset 3: evaluation of the conducted measures

- Dataset 4: participant observations

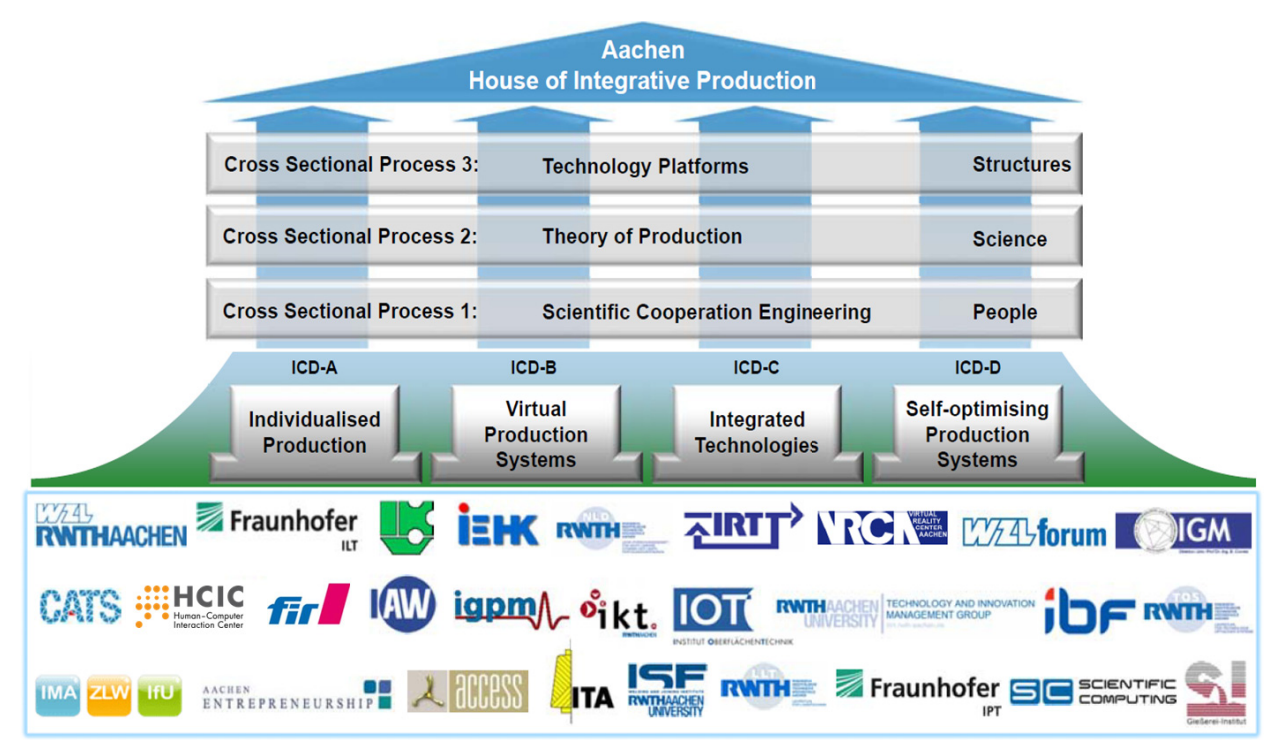

Figure 2. Structure of the CoE with its four Integrative Cluster Domains (ICDs) and three Cross Sectional Processes (CSPs) 
This characteristic provides the advantage that a holistic view on the object of investigation can be obtained, since it is observed from various perspectives [cf. Lamnek 1995 in 24]. In order to establish access to the data as well as to identify patterns several procedural steps of analysis have been conducted:

- As a first step, different phenomena of interdisciplinary collaboration are worked out that result from the researchers' perception.

- In another step of the analysis, critical factors (critical incidents - CIs) - in reference to the Critical Incidents Technique [25] - are identified from each dataset.

- After having empirically reasoned a theoretical saturation 30 CIs have been transferred to three patterns.

Through these three steps we are able to discuss the topic in a broader way. We have no indication, however, that our single case represents general CIs of IDR. But there are other empirical outcomes that corroborate our findings. These findings and all results are elaborately presented and discussed in "Interdisciplinary Research collaboration: Critical Incidents from the researchers" perspective“" [1]. In this paper we focus on one selected pattern that deals with the elaboration of an integrated knowledge management with respect to reaching the common interdisciplinary vision. This is presented in the next section.

\section{Results for Integrative Knowledge Management}

Our research activities aimed at empirically exploring how researchers involved in the CoE perceive this mode of research. The research was also supposed to investigate what they need to challenge this mode of research. To reach an interdisciplinary integration during the research process the researchers involved demanded an integrative knowledge management. Its importance is shown in its support to accomplish the common interdisciplinary vision. The researchers involved demanded the continuous support of synchronization and adaptation of the individual research activities with regard to this vision. In this context, integrative describes the assumption that a combination of different supportive measures is of importance.

As far as the researchers' perception of interdisciplinary collaboration is concerned, it cannot be conducted by the mere addition of researchers from various disciplines. Hence, a continuous initiation of a project-, level-, and discipline-extensive networking is considered to be crucial. With regard to the common vision, an identification of interfaces is required, which, however, have to be continuously edited by means of an interactive exchange. It is also assumed that regular meetings are necessary to enable networking. In this context, it is important to provide a sufficient allocation of time for both the content-related negotiation processes, e.g. with regards to the common vision, and the social networking. The researchers involved also have to use these dates to meet and learn to appreciate each other in order to successfully work together in a team. Moreover, the researchers ascribe a significant role to so-called key persons who, however, are only capable of pushing ahead the active networking if a constant exchange between them and a reflection of research findings take place. The key persons are also supposed to have an overview on the contents and they need to be aware of their role. Furthermore, the researchers involved need a high motivation and willingness to participate in interdisciplinary collaborations. Both motivation and willingness can be supported by incentives, which is in line with the demands. In order to work on the common interdisciplinary vision, it is of importance to raise awareness for it. This raise of awareness can be supported by the researchers' demand for a visualization. With it, it is possible to localize researchers and projects within an overall image, which helps to identify and further process interfaces. Beyond that, it enables the researchers to consciously recognize and know their position and contribution to the overall goal.

A technical support is claimed to be necessary by the researchers involved, as the challenges to successful communication increase with working in an interdisciplinary collaboration. This technical support can, for instance, be realized by implementing a clearly structured, central communication platform. This technical, particularly virtual support is supposed to exceed a data management system. Furthermore, both a communication and cooperation platform and the visualization of the networking are considered to be important measures to handle staff turnover and to support the flow of communication and cooperation.

This demand of integrative knowledge management by the researchers of the CoE can be considered as being genera. But their elaboration is specific to each cluster and has to take place in close coordination with the respective needs of the involved researchers. To demonstrate how we deal with the virtual support within the $\mathrm{CoE}$ we present our Scientific Cooperation Portal in the following, which is jointly developed by the team of the CSPs.

\section{SUPPORTING INTEGRATTIVE KNOWLEDGE MANAMGENT BY TECHNICAL MEANS}

The so-called Scientific Cooperation Portal is comprised of various apps, ranging from project management support to target-group-specific implementations. Based on the findings mentioned in chapter II. these apps have in common that they foster information dissemination on different levels within the CoE [15] and therefore support an integrative knowledge management by the connection of various information sources [26]. Thus, management support is given by basic features, e.g. a news section, a publication database and a calendar application.

More individualized aspects are addressed by a web app, which enables the user to identify interfaces between entities (e.g. projects or other persons) in the research cluster. This mainly aims at two identified CIs of IDR: The focus on communication and terminology as well as the identification and visualizing of connections [1]. These connections between entities are based on the usage of terminologies in this example: e.g. if two projects have a given set of terminologies in common, a connection between these two projects is displayed in the web application.

On the basis of this example a three step concept is outlined to address this idea: On the one hand the extraction of terminologies has to be discussed. On the other hand the mapping of entities has to be realized. The last step represents the visualization of the connection mentioned above. 


\section{A. Extraction of terminologies}

The first step in the identification process of connections can be seen in the extraction of terminologies from a given data source. As publications of all projects are stored in a common database on the Scientific Cooperation Portal, a process is necessary that allows the web application to access these publications and extract terminologies from the data source.

As Text Mining can be described as "a range of technologies for analyzing and processing semi-structured and unstructured text data" [27] this methodology can serve as one possible solution for the above mentioned challenge. After various pre-processing steps, including e.g. the tokenization of the publications as well as the filtering of stop words, POS-tagging is used to derive nouns from the publications as these word types are considered as main source for terminologies in the CoE. After that a standard tf-idf algorithm is applied to determine the frequencies of words within the publications. This aims at a vectorial description of the publications, which can be used in further processes. By combining several publications, which e.g. have been issued in the context of a project, a vectorial description of an entity within the research cluster becomes possible.

Within this vectorial description of frequencies various challenges concerning the linguistic properties of the terminologies have to be addressed. For example, so-called collocations are a typical challenge. These can be described as phrases which are considered to have an existence beyond the sum of the parts. This includes compounds (disk drive) or stock phrases (bacon and eggs) [28]. With this in mind the given publications are analyzed not only focusing the frequencies of words but also regarding words that are often used in combinations.

\section{B. Mapping of Entities}

After a vectorial description of terminologies has been created the second step represents the mapping of entities in order to derive statistically based connections. By using classification algorithms "the task is to classify a given data instance into a pre-specified set of categories" [29]. With the regard to the last chapter the pre-specified set of categories can been seen in the different entities (e.g. projects) in the $\mathrm{CoE}$, which are described by the combined vectors of several publications. The web application then extracts terminologies from e.g. a new publication and uses a cluster algorithm to determine how likely this publication fits into the set of categories. The result is a set of probabilities, which can be used to describe the distance of a publication to the set of categories.

This leads to another thought in the context of distance between terminologies: the semantic similarity of words. The acquisition of meaning by automated systems is quite a challenge in Natural Language Processing. Approaches in this context focus mainly on a relative measure for semantic similarity, which can be used to determine how similar a word is to known words [28]. Thus, the relative measure enables the web application to classify the meaning of words by the use of vector space measures and binary similarity measures (e.g. Jaccard coefficient).

These similarity measures serve as mathematical method to describe the semantic meaning of a word in relation to other words. This is based on the analysis of frequency matrixes, which contain information about the context in which a word is used. Different matches e.g. between two contexts lead to higher ranking of semantic meaning between two words and therefore allow the web application to link these two words.

\section{Visual data analysis}

The third step in the concept of the web application is the visualization of the above mentioned results. The main goal is to depict new connections for the user on the basis of common terminologies and, therefore, common (research) topics between the user's project and other entities in the CoE. Hence, the visualization has to map two major aspects:

- The extracted terminologies from the user's project to the terminologies of other projects.

- The semantic similarity of words in relation to other words used in the terminologies of the CoE.

The first issue can be addressed by a metric that is based on the probabilities as described in section B of this chapter. As a consequence the probabilities serve as a measure of distance between entities in the CoE: If the classification reveals a higher probability, the depicted distance is closer and vice versa.

As the semantic similarity is represented by relative measures of one word in relation to another word (cf. in section B of this chapter), this aspect has also to be realized in the visualization. Therefore, this visualization has to depict word-to-word connections with regard to the relative measure of semantic similarity. One possible solution is a so-called tree graph (cf. Fig. 3).

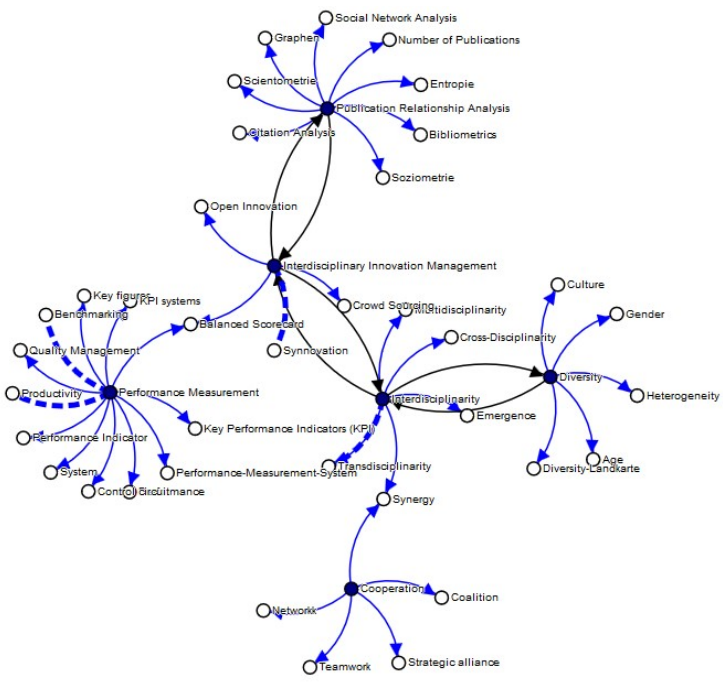

Figure 3: Example of a tree graph used in the web application

This tree graph allows the user to detect easily in how far a semantic similarity persists between its own terminologies and the terminologies used in other projects. Following this idea one goal of visual analytics, the synthesis of information and to communicate this assessment effectively for action is fulfilled [30]. Further aspects include separate pages for each terminology in a Wikipedia style: key-persons (e.g. experts) can contribute to the added-value of the application by integrating their knowledge in definitions and the recommendation of further sources (e.g. literature). The next step can be seen in further exchange on acquiring a common understanding of terminologies (e.g. workshops). 
In this context the web application addresses the demands outlined in chapter II. On a shallow level the visualization part of the app enables the researchers to be aware of other activities within the CoE. Key-persons for each terminology become identifiable and potential synergies visible. These can be used to foster further exchange processes regarding the semantic differentiation of terminologies. Going more into detail, the visualization can be seen as the individual part of the researchers' contribution to the vision of the CoE. As the depicted terminologies represent the researchers' field of action the visualization of connections show the embedding in related fields. Therefore, this allows the researcher to recognize his placing within the $\mathrm{CoE}$ and the placing of his field of action in an overall context.

\section{EXPERIENCES AND OUTLOOK}

The selected results give some indication of which aspects of interdisciplinary collaboration are important from the researchers' point of view to support the expected integration. Moreover, IDR is associated with high expectations, since synergies are supposed to be achieved. With regard to the common vision they demand a visualization since it is possible to localize researchers and projects within an overall image. Beyond that, the data have shown that it is essential to facilitate the expediting of the content-related networking by key persons. It is crucial for the key persons to be conscious about their role and that they are correspondingly promoted.

All these results underline the consensus on the insufficiency of merely adding researchers from various disciplines to make a research effort interdisciplinary. Therefore, integrative knowledge management has to be evaluated in a process-accompanying manner. In order to reinforce the findings it may be important to examine further research clusters to explore whether similar patterns can be identified. Additionally, to gain more insights into how CIs may vary in different scientific fields represents another important starting point for further research. The subsequent step is a quantification of the CIs. This aims at the development of quantified correlations between current qualitative statements.

As the web application is currently in conceptual stage the technical proof of concept has yet to be done. The major challenge behind this can be seen in the investigation of technical parameters, which enable the application to derive meaningful data from the Scientific Cooperation Platform database. If this has been finalized following steps will include support measures for the CoE researchers in order evaluate and stabilize the results shown in the application on a semantical level (like workshops etc.). On a technical level subsequent steps will include experiments with clustering algorithms in order to reveal thematic groups based on publication within the $\mathrm{CoE}$, which are yet not revealed.

\section{ACKNOWLEDGMENT}

The authors would like to thank the German Research Foundation DFG for the kind support within the Cluster of Excellence "Integrative Production Technology for HighWage Countries".

\section{REFERENCES}

[1] C. Jooß, "Interdisciplinary Research collaboration: Critical Incidents from the researchers" perspective," in Gestaltung von Ko- operationsprozessen interdisziplinärer Forschungsnetzwerke, Norderstedt: BOD-Verlag, pp. 146-179, 2014, pp. 10 ff., p. 177, p. 169, p. 149 , p. 97

[2] J. A. Jacobs and S. Frickel, "Interdisciplinarity: A Critical Assessment," Annual Review of Sociology, vol. 35, pp. 43-65, p. 47f., 2009.

[3] J. T. Klein, Interdisciplinarity: history, theory, and practice, Detroit: Wayne State University Press, 1990.

[4] D. Rhoten and S. Pfirman, "Women in interdisciplinary science: Exploring preferences and consequences," Research Policy, vol. 36 , no. 1 , pp. $56-75,2007$, p. 58 , p. 57.

[5] F. J. Van Rijnsoever and L. K. Hessels, "Factors associated with disciplinary and interdisciplinary research collaboration," Research Policy, vol. 40, no. 3, p. 463-472, April 2011, p. 463 f.

[6] M. Gibbons, C. Limoges, H. Nowotny, S. Schwartzman, P. Scott, and $\mathrm{M}$. Trow, The new production of knowledge: the dynamics of science and research in contemporary societies, London: Sage, 1994.

[7] National Academies (U.S.), Committee on Facilitating Interdisciplinary Research, Committee on Science, Engineering, and Public Policy (U.S.), National Academy of Sciences (U.S.), and National Academy of Engineering; Institute of Medicine (U.S.), Eds., Facilitating interdisciplinary research, Washington, D.C: National Academies Press, 2005, p. 16, 27.

[8] S. W. Aboelela et al., "Defining Interdisciplinary Research: Conclusions from a Critical Review of the Literature," Health Services Research, vol. 42, no. 1, pp. 329-346, 2007, p. 340.

[9] A. L. Porter and I. Rafols, "Is science becoming more interdisciplinary? Measuring and mapping six research fields over time," Scientometrics, vol. 81 , no. 3, pp. 719-745, April $16^{\text {th }} 2009$, p. 720.

[10] R. Defila and A. Di Giulio, "Interdisziplinarität und Disziplinarität. Zwischen den Fächern über den Dingen? Universalisierung versus Spezialisierung akademischer Bildung," in Zwischen den Fächern - über den Dingen?, J. H. Olbertz, Ed., Opladen: Leske und Budrich, 1998, pp. 11-127, p. 114.

[11] G. Strina, "Zur Messbarkeit nicht-quantitativer Größen im Rahmen unternehmenskybernetischer Prozesse," unpublished, p. 29.

[12] G. Melin, "Pragmatism and self-organization: Research collaboration on the individual level," Research Policy, vol. 29, no. 1, pp. 31-40, January 2000, p. 32.

[13] C. Brecher, Integrative Production Technology for High-wage Countries, Berlin, Heidelberg: Springer-Verlag, 2012. http://dx.doi.org/10.1007/978-3-642-21067-9

[14] Cluster of Excellence 'Integrative Production Technology for High-Wage Countries' Renewal Proposal for a Cluster of Excellence - Excellence Initiative by the German Federal and State Governments to Promote Science and Research at German Universities, unpublished.

[15] T. Vaegs et al., "Enhancing Scientific Cooperation of an Interdisciplinary Cluster of Excellence via a Scientific Cooperation Portal," Proceedings of the ICELW 2014, New York, June $11^{\text {th }}-13^{\text {th }}$.

[16] C. Jooß et al., "Scientific Cooperation Engineering in the Cluster of Excellence Integrative Production Technology for High-Wage Countries at RWTH Aachen University," accepted at 5th International Conference of Education, Research and Innovation (ICERI) 2012.

[17] N. Carayol and T. U. N. Thi, "Why do academic scientists engage in interdisciplinary research?," Research Evaluation, vol. 14, no. 1 , pp. 70-79, 2005. $\quad$ http://dx.doi.org/10.3152/14715440 5781776355

[18] A. F. J. van Raan, "Scientometrics: State-of-the-art," Scientometrics, vol. 38, no. 1, pp. 205-218, January 1997. http://dx.doi.org/10.1007/BF02461131

[19] D. Rhoten, "Interdisciplinary Research: Trend or Transition," Items \& Issues, vol. 5, no. 1-2, pp. 6-11, 2004.

[20] A. L. Porter, J. D. Roessner, A. S. Cohen, M. Perreault, "Interdisciplinary research: meaning, metrics and nurture," Research Evaluation, vol. 15, no. 3, pp. 187-195, 2006. http://dx.doi.org/10.3152/147154406781775841

[21] C. Raasch, V. Lee, S. Spaeth, C. Herstatt, "The rise and fall of interdisciplinary research: The case of open source innovation," 
Research Policy, vol. 42, no. 5, pp. 1138-1151, June 2013. http://dx.doi.org/10.1016/j.respol.2013.01.010

[22] M. M. Millar, "Interdisciplinary research and the early career: The effect of interdisciplinary dissertation research on career placement and publication productivity of doctoral graduates in the sciences," Research Policy, vol. 42, no. 5, pp. 1152-1164, June 2013, p. 1163.

[23] J. Kruse, Reader: Einführung in die Qualitative InterviewForschung, Freiburg, 2011, p. 12.

[24] U. Kelle, Die Integration qualitativer und quantitativer Methoden in der empirischen Sozialforschung. Theoretische Grundlagen und methodologische Konzepte, $2^{\text {nd }}$ ed., Wiesbaden: VS Verlag für Sozialwissenschaften, 2008, p. 50. http://dx.doi.org/10.1007/978-3531-91174-8

[25] J. C. Flanagan, "The critical incident technique," Psychological Bulletin, vol. 51, no. 4, pp. 327-358, 1954 http://dx.doi.org/10.1037/h0061470

[26] H. Krcmar, Einführung in das Informationsmanagement, Berlin, Heidelberg: Springer, 2011. http://dx.doi.org/10.1007/978-3-64215831-5

[27] G. Miner, D. Delen, J. Elder, A. Fast, T. Hill, and R. A. Nisbet, "The Seven Practice Areas of Text Mining," Practical Text Mining and Statistical Analysis for Non-structured Text Data Applications, Waltham, Oxford: Academic Press, 2012, pp. 29-41.

[28] C. D. Manning and H. Schütze, Foundations of Statistical Natural Language Processing, Cambridge, Massachusetts, London: MIT Press, 2003.

[29] R. Feldman and J. Sanger, The Text Mining Handbook - Advanced Approaches in Analyzing Unstructured Data, Cambridge: Cambridge University Press, 2006. http://dx.doi.org/10.1017/ CBO9780511546914

[30] D. Keim, J. Kohlhammer, G. Ellis, and F. Mansmann, Mastering the Information Age Solving Problems with Visual Analytics, Goslar: Thomas Müntzer, 2010.

\section{AUTHORS}

Dr. Claudia Jooß is researcher and leader of the research group "Knowledge Engineering" at the Center for Learning and Knowledge Management (ZLW) in the institute cluster IMA/ZLW \& IfU at RWTH Aachen University, Aachen, Germany (e-mail: claudia.jooss@imazlw-ifu.rwth-aachen.de).

Thomas Thiele is researcher in the research group "Knowledge Engineering" at the Center for Learning and Knowledge Management (ZLW) in the institute cluster IMA/ZLW \& IfU at RWTH Aachen University, Aachen, Germany (e-mail: thomas.thiele@ima-zlw-ifu.rwthaachen.de).

Dr. Rene Vossen is managing director of the Ass. Institute for Management Cybernetics (IfU) in the institute cluster IMA/ZLW \& IfU at RWTH Aachen University, Aachen, Germany (e-mail: rene.vossen@ima-zlw-ifu. rwth-aachen.de).

Prof. Anja Richert is managing director of the Center for Learning and Knowledge Management (ZLW) in the institute cluster IMA/ZLW \& IfU at RWTH Aachen University, Aachen, Germany (e-mail: anja.richert@ima-zlwifu.rwth-aachen.de).

Prof. Sabina Jeschke is director of the institute cluster IMA/ZLW \& IfU and vice dean of the department of mechanical engineering at RWTH Aachen University, Aachen, Germany (e-mail: sabina.jeschke@ima-zlwifu.rwth-aachen.de).

This article is an extended and modified version of a paper presented at the International Conference on E-learning in the Workplace 2015 (ICELW'15), held in June 2015, at Columbia University in New York, NY, USA. Submitted 28 July 2015. Published as resubmitted by the authors 30 August 2015. 